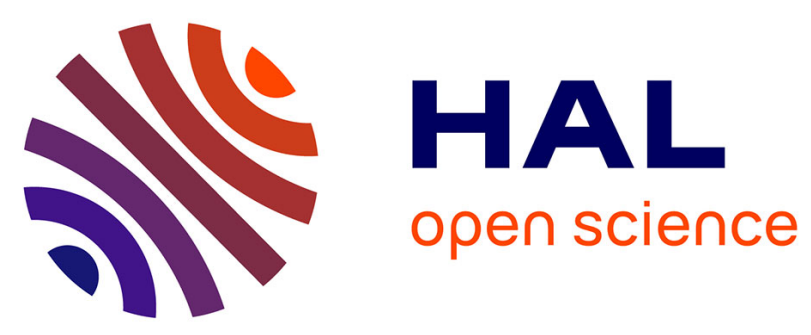

\title{
Comprehension of peculiar local emission behavior of InGaAs quantum well by colocalized nanocharacterization combining cathodoluminescence and electron microscopy techniques
}

Joyce Roque, Georges Beainy, Névine Rochat, Nicolas Bernier, Sylvain David, Jeremy Moeyaert, Mickaël Martin, Thierry Baron, Jean-Luc Rouvière

\section{To cite this version:}

Joyce Roque, Georges Beainy, Névine Rochat, Nicolas Bernier, Sylvain David, et al.. Comprehension of peculiar local emission behavior of InGaAs quantum well by colocalized nanocharacterization combining cathodoluminescence and electron microscopy techniques. Journal of Vacuum Science \& Technology B Microelectronics and Nanometer Structures, 2018, 36 (4), pp.042901. 10.1116/1.5033363 . hal-01947865

\section{HAL Id: hal-01947865 \\ https://hal.univ-grenoble-alpes.fr/hal-01947865}

Submitted on 6 Apr 2020

HAL is a multi-disciplinary open access archive for the deposit and dissemination of scientific research documents, whether they are published or not. The documents may come from teaching and research institutions in France or abroad, or from public or private research centers.
L'archive ouverte pluridisciplinaire HAL, est destinée au dépôt et à la diffusion de documents scientifiques de niveau recherche, publiés ou non, émanant des établissements d'enseignement et de recherche français ou étrangers, des laboratoires publics ou privés. 


\title{
Comprehension of peculiar local emission behavior of InGaAs quantum well by colocalized nanocharacterization combining cathodoluminescence and electron microscopy techniques
}

\author{
Joyce Roque, ${ }^{\text {a) }}$ Georges Beainy, Névine Rochat, and Nicolas Bernier \\ Univ. Grenoble Alpes, CEA, LETI, F-38000 Grenoble, France \\ Sylvain David, Jérémy Moeyaert, Mickael Martin, and Thierry Baron \\ Univ. Grenoble Alpes, CNRS, CEA/LETI Minatec, LTM, F-38054 Grenoble Cedex, France \\ Jean-Luc Rouvière \\ Univ. Grenoble Alpes, CEA, INAC, F-38000 Grenoble, France
}

(Received 5 April 2018; accepted 12 June 2018; published 2 July 2018)

\begin{abstract}
The electronic and structural properties of an $\operatorname{In}_{\mathrm{x}} \mathrm{Ga}_{1-\mathrm{x}} \mathrm{As}$ quantum well (QW) stacking between AlAs barriers grown on $300 \mathrm{~mm}$ (001) silicon substrate by metalorganic chemical vapor deposition were investigated. Nanometer scale and spatially colocalized characterization combining low temperature cathodoluminescence $(\mathrm{CL})$ and scanning transmission electron microscopy was performed. The combined interpretation of luminescence and strain measurement provides an exhaustive landscape of such complex sample. Particularly, CL analysis highlights luminescent regions characterized by quasicircular shapes and a peculiar optical emission consisting of a double peak. The characterizations provide a comprehensive analysis of these specific luminescence features. These luminescent regions, detected all over the sample, seem to be correlated to local increases in carbon and indium content in AlAs barriers and in the InGaAs QW, respectively, induced by local strain variations. These modifications alter InGaAs QW properties and thus its optical emission efficiency. Published by the AVS. https://doi.org/10.1116/1.5033363
\end{abstract}

\section{INTRODUCTION}

Epitaxial growth of III-V materials on $300 \mathrm{~mm}$ (001) silicon (Si) substrates by metal organic chemical vapor deposition (MOCVD) is a promising approach for future industrial integration of both optoelectronic and photovoltaic devices. Heteroepitaxy allows to benefit simultaneously from both the silicon technology developed by microelectronic industry and the unique optical functionality of III-V materials. Unfortunately, the III-V epitaxial layers grown on Si suffer from the appearance of numerous structural defects. One particularly striking example is that of GaAs where its lattice and thermal mismatch with $\mathrm{Si}$, in addition to its polar material property, induce a large amount of complex defects such as antiphase boundaries, stacking faults, or dislocations. ${ }^{1,2}$ Recent studies have demonstrated a possibility of annihilating antiphase boundaries with very low rates of stacking faults for 2D GaAs layer growth, ${ }^{3,4}$ by annealing $\mathrm{Si}$ substrates under hydrogen atmosphere. Nonetheless, a high dislocation density is still present despite the addition of a $400 \mathrm{~nm}$ GaAs buffer layer. Such thickness is not efficient enough to fully annihilate dislocations. Thus, numerous threading dislocations (TD) can still reach and cross the $\mathrm{In}_{\mathrm{x}} \mathrm{Ga}_{1-\mathrm{x}} \mathrm{As}$ quantum well (QW). These local defects dramatically impact the active nanostructure efficiency by changing its electrical and optical properties. In this study, the $\mathrm{In}_{\mathrm{x}} \mathrm{Ga}_{1-\mathrm{x}} \mathrm{As} \mathrm{QW}$ is embedded between two AlAs barriers. AlAs has been chosen to reduce surface segregation of

$\overline{{ }^{a} \text { Electronic mail: joyce.roque@cea.fr }}$ indium atoms at QW interfaces frequently observed in InGaAs/GaAs structures. ${ }^{5}$

The electronic band structure of such InGaAs QWs heteroepitaxial growth is known to be affected by many factors. First, indium quantity governs band gap energy by following the Vergard's law. ${ }^{6}$ Second, carrier confinement in the QW induces band gap modifications depending mainly of QW thickness and barrier characteristics. Third, heteroepitaxial growth induces strain that affects the electronic properties of semiconductors. In 2D heteroepitaxial growth, biaxial strain must be taken into account to determine the energy emission. ${ }^{7}$ In our case, the InGaAs QW will be mainly strained by the GaAs buffer layer. Fourth, the epitaxial layers suffer from the incorporation of impurities which can modify radiative luminescence by adding electronic levels within the band gap. ${ }^{8}$ All these parameters commonly affect the electronic properties of InGaAs QW. In addition, due to the growth on Si substrate, the existence of dislocations is able to induce strong and local modifications in $2 \mathrm{D}$ planes such as strain variations, indium fluctuations, ${ }^{9}$ thickness, and impurities concentration variations which lead to important spatial variations of QW emission.

Accordingly, this study conducts an in-depth spatial correlation between strong optical modifications and structural properties using cathodoluminescence (CL) and scanning transmission electron microscopy (STEM or TEM). Complex hyperspectral CL cartography is used to select a local peculiar luminescent feature detected on the sample surface. The area of interest is then extracted precisely and analyzed by transmission electron microscopy in cross section to understand the correlation between peculiar optical 
properties and structural characteristics. To spatially correlate both characterizations, an experimental method has been developed ${ }^{10}$ and improved to study specific emission zones.

\section{EXPERIMENTAL PROCEDURE}

\section{A. Sample studied}

The growth of III-V layers was performed with organometallic source materials in an Applied Materials MOCVD reactor configured for $300 \mathrm{~mm}$ diameter $\mathrm{Si}$ (001) substrates. Before growth, the $\mathrm{Si}$ substrate was deoxidized in a SiConi ${ }^{\mathrm{TM}}$ chamber using a $\mathrm{NF}_{3} / \mathrm{NH}_{3}$ remote plasma. ${ }^{3}$ This wafer then undergoes a thermal annealing under hydrogen atmosphere at high temperature to structure atomic steps at the surface. The grown layers stack is detailed in Fig. 1. First, a GaAs buffer layer (GaAs No. 1) is grown on $\mathrm{Si}$ with a two-step process: (1) a first nucleation layer of around $30 \mathrm{~nm}$ grown at low-temperature $\left(350^{\circ} \mathrm{C}\right)$; $(2)$ a second layer grown at a high-temperature $\left(600{ }^{\circ} \mathrm{C}\right)$ for a total thickness of $300 \mathrm{~nm}$. Two thick and intermediate layers, AlAs layer $(55 \mathrm{~nm})$ and GaAs layer (GaAs No. 2, $100 \mathrm{~nm})$, have been grown on the GaAs buffer layer No. 1. Finally, an $\mathrm{In}_{0.1} \mathrm{Ga}_{0.9}$ As quantum well $(6 \mathrm{~nm})$ is grown embedded between two AlAs barriers (Nos. 1 and 2, $10 \mathrm{~nm}$ ). To protect upper layers from oxidation, a GaAs capping layer $(30 \mathrm{~nm})$ has been deposited.

\section{B. Experimental details}

To investigate the optical properties, the sample is studied in top view by CL at a low temperature $(10 \mathrm{~K})$, thanks to the use of Attolight CL equipment. The electron beam of the scanning electron microscope (SEM) is used at $5 \mathrm{keV}$ to excite luminescence which is collected with an in-axis reflective optical collection system. The optical signal is analyzed by a spectrophotometer (150 lines/mm grating blazed at $500 \mathrm{~nm}$ ) and detected on a CCD. By scanning electron beam on the sample, a hyperspectral image is formed and recorded, which means that each pixel of the CL cartography corresponds to a luminescence spectrum. This hyperspectral image is then treated by fitting each local spectrum using a sum of Gaussian peaks corresponding to each component of the luminescence spectrum. The position and intensity mappings of each fitted component are used to characterize local variations of InGaAs QW emission.

The structural properties, such as nature, position of defects, or variation of well width, were characterized in cross-section by STEM based on a field-emission-gun (FEG) electron source. Thin lamellas are prepared with a FIB-SEM tool (Helios NanoLab 450S from FEI) combining focused ion and electron beam. First, the sample is locally covered by carbon and platinum $(\mathrm{Pt})$ layers using gaseous precursors deposited under the electron beam in order to protect it from Ga beam damages. Second, to have sufficient thickness during etching process, a platinum layer using the ion beam is deposited. The sample is extracted using a focused ion beam and etched to realize cross section lamella. Additional TEM and STEM images were performed in a TEM Titan Ultimate

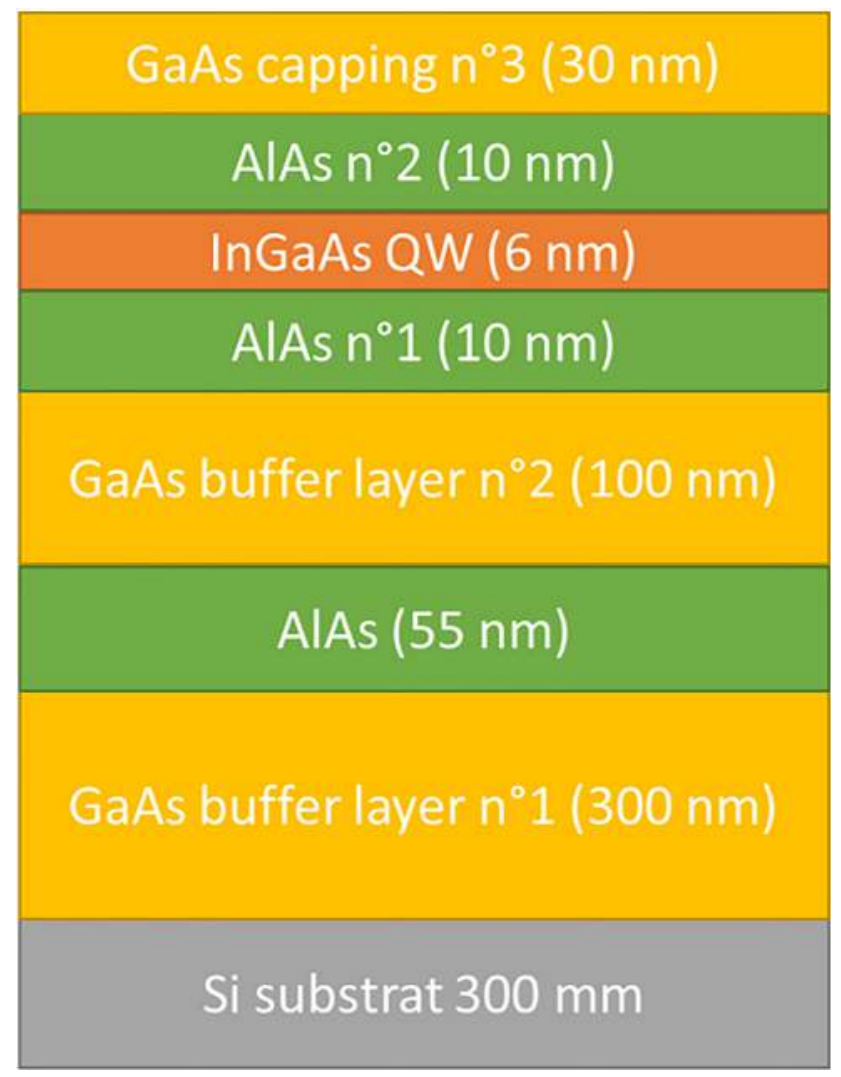

FIG. 1. (Color online) Sketch of III-V layers stacking grown on the silicon substrate.

and TEM Tecnai, respectively, with an incident beam energy of $200 \mathrm{keV}$.

To understand the influence of emergent defects on the QW optical properties, spatially correlated CL and STEM images have been performed to associate the optical and morphological properties. For this purpose, a specific experimental method ${ }^{10}$ is performed by identifying areas of interest obtained with $\mathrm{CL}$ and then by extracting the corresponding region for STEM analyses. As this technique involves the use of FIB-SEM, the preparation of thin lamellas for STEM and TEM images is always performed after top view CL studies are directly realized on bulk samples. This avoids additional damages, which can act as nonradiative recombination center.

Perfect localization on the surface at nanoscale is particularly challenging on 2D layers. To overcome this problem, a first step consists in marking the sample surface for top view $\mathrm{CL}$ experiments. It is realized by platinum marks deposited by precursor gas using the electron-beam in the FIB-SEM. Thus, some triangular Pt marks of $250 \mathrm{~nm}$ width and $300 \mathrm{~nm}$ height ${ }^{10}$ are grown on the surface. A second step of carbon marks is carried out after CL experiments on specific luminescent areas previously observed. These are chosen to be easy to identify under Pt capping of lamellas. Carbon marks are deposited as Pt ones and measure typically $100 \times 200 \mathrm{~nm}$ with $30 \mathrm{~nm}$ height. These new carbon marks allow accurate TEM lamella extraction and thinning at the exact location of areas of interest. 

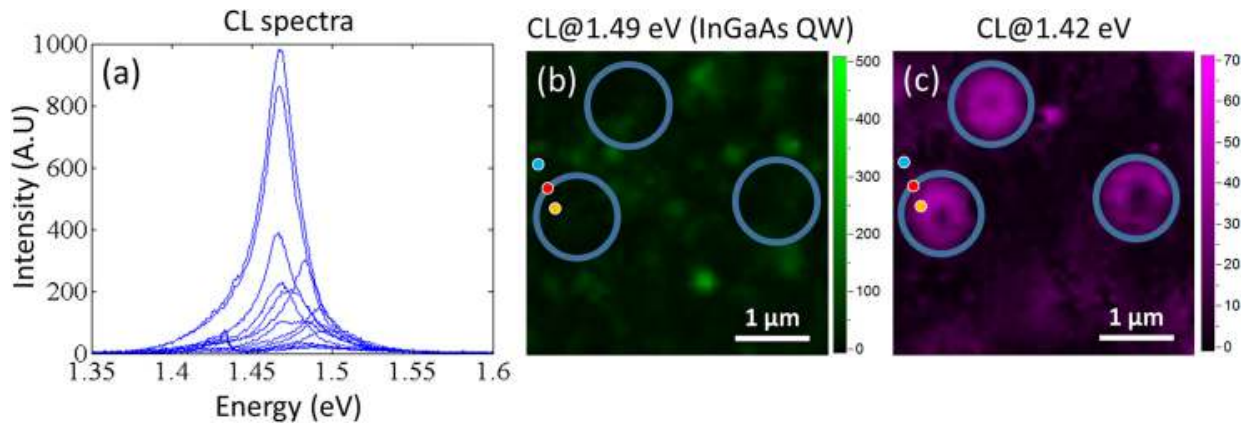

FIG. 2. (Color online) (a) Typical spectra measured in CL mapping. CL intensity mapping (expressed in arbitrary unit) (b) at $1.49 \mathrm{eV}$ (emission of InGaAs $\mathrm{QW}$ ) and (c) at $1.42 \mathrm{eV}$. Circles highlight peculiar optical emission detected at $1.42 \mathrm{eV}$ on mapping (c). Mapping (b) shows there is no corresponding optical variations at $1.49 \mathrm{eV}$.

To understand the impact of structural defects, nanobeam precession electron diffraction (N-PED) ${ }^{10-12}$ method has been used to estimate local crystal distortions. In contrast with standard nanobeam electron, which uses a nearly parallel beam resulting in a spot size of $\sim 5-6 \mathrm{~nm}$, nanobeam precession uses a convergent beam (typically around $3 \mathrm{mrad}$ ) resulting in a smaller spot size of about $2-3 \mathrm{~nm}$. The electron beam is precessed by using the STEM scan coils to obtain more uniform and numerous diffraction spots. N-PED has been acquired on a FEI Titan Themis microscope equipped with an X-FEG source operated at $200 \mathrm{keV}$, and recorded on a $2000 \times 2000$ Gatan CCD camera. Characterization estimates local crystal strains $\varepsilon_{\mathrm{xx}}, \varepsilon_{\mathrm{zz}}$ along [110] and [001] directions, respectively, with nanoscale spatial resolution. For this purpose, a $200 \mathrm{~nm}$ thick specimen has been made to avoid strain relaxation. In addition to N-PED results, geometrical phase analysis (GPA) method $^{13-15}$ is used. It allows to measure strain from high resolution scanning transmission electron microscopy (HRSTEM) images. ${ }^{9}$ An HR-STEM lattice image is characterized by the presence of strong components in its Fourier transform. By performing an inverse Fourier transform, phase components give information about local displacements of atomic planes in the plane of images. The field can be derived by applying the method to two noncollinear Fourier components. Local strain components can be found by analyzing the derivative of the displacement field. GPA is less sensitive $\left(3 \times 10^{-3}\right)$ than N-PED $\left(1 \times 10^{-4}\right)$, but it is more spatially resolved to observe local variations at the nanoscale. HR-STEM images have been done with the FEI Titan Themis microscope with the same conditions which were stated earlier.

Time-of-flight secondary ion mass spectroscopy (ToFSIMS) is performed to estimate indium quantity contained in the InGaAs QW and to detect impurity presence. ToF-SIMS

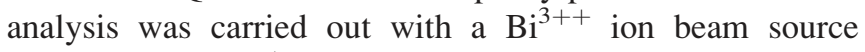
$(25 \mathrm{keV})$ and a $\mathrm{Cs}^{+}$ion beam for sputtering $(1 \mathrm{keV})$ using a ToF-SIMS V from ION TOF. The ion bombardment was performed at a $45^{\circ}$ incidence angle, and the beam raster size was defined as $300 \times 300 \mu \mathrm{m}^{2}$.

\section{RESULTS AND DISCUSSION}

\section{A. Cathodoluminescence study}

The study of InGaAs QW emission by low temperature CL $(10 \mathrm{~K})$ highlights complex emission spectra (Fig. 2).
Important intensity and width variations combined with strong energy shift are detected on the scanned surface [Fig. 2(a)]. QW free-exciton (FE) is the main luminescent line detected on the CL image. It is ranging between 1.463 and $1.498 \mathrm{eV}$ and is randomly detected on the sample surface [Fig. 2(b)] whereas other particular spectral contributions at $1.42 \mathrm{eV}$ exhibit a circular shape [Fig. 2(c)]. These particular luminescent circles have a $1 \mu \mathrm{m}$ diameter with quenching spots present in the center of all of them. These features are present on the sample with a density of $\sim 9 \times 10^{6} \mathrm{~cm}^{-2}$, which means an average of three circles of $1 \mu \mathrm{m}$ diameter on $6 \times 6 \mu \mathrm{m}^{2}$.

This FE energy is usually used to determine QW indium concentration. For this purpose, simulation of electronic properties has been performed with NEXTNANO software ${ }^{16}$ by using an $\operatorname{In}_{\mathrm{x}} \mathrm{Ga}_{1-\mathrm{x}} \mathrm{As} \mathrm{QW}$ of $6 \mathrm{~nm}$ thickness confined between two AlAs barriers of $10 \mathrm{~nm}$ thickness, biaxially strained on relaxed GaAs at $0 \mathrm{~K} .{ }^{17}$ Since the GaAs layer No. 2 is far from the $\mathrm{Si}$ substrate, a first approximation is to assume that the GaAs layer close to QW is not more strained by $\mathrm{Si}$. By taking into account exciton binding energy $\mathrm{E}^{\mathrm{ex}}=13 \mathrm{meV}$ and valence band degeneracies induced by biaxial compressive strain on the QW, an In content of $11 \%$ $\pm 1.5 \%$ (Table I) was estimated for quantum confinement of the lowest electron and heavy-hole sublevels transition.

The determined In concentration is in good agreement with that obtained by ToF-SIMS analysis $(12.3 \% \pm 1.2 \%)$. The tiny difference can be explained by local In fluctuations, QW thickness, and strain variations caused by all defects. ${ }^{2,18}$ Moreover, ToF-SIMS is not a local measurement contrary to CL.

Figure 3 highlights evolution of CL spectra for three different areas located outside, at the edge, and inside a circular

TABLE I. Variation of the gap (e-hh transition) and free-exciton transition (FE) for different indium concentrations, with exciton binding energy defined by $\mathrm{E}^{\mathrm{ex}}=13 \mathrm{meV}$. Results obtained by NextNano simulations of strained $\mathrm{In}_{\mathrm{x}} \mathrm{Ga}_{1-\mathrm{x}} \mathrm{As} \mathrm{QW}$ on GaAs, for $6 \mathrm{~nm}$ QW thickness, between two AlAs barriers of $10 \mathrm{~nm}$ thicknesses, at $0 \mathrm{~K}$. FE emission values and the Indium quantities obtained by simulation corresponding to FE emission values detected on CL image are in boldface.

\begin{tabular}{lccccc}
\hline \hline $\operatorname{In}(\%)$ & $\mathbf{1 0}$ & $\mathbf{1 1}$ & $\mathbf{1 2}$ & $\mathbf{1 3}$ & 14 \\
\hline $\mathrm{Eg}(\mathrm{eV})$ & 1.5066 & 1.4956 & 1.4847 & 1.4736 & 1.4631 \\
$\mathrm{FE}(\mathrm{eV})$ & $\mathbf{1 . 4 9 3 6}$ & $\mathbf{1 . 4 8 2 6}$ & $\mathbf{1 . 4 7 1 7}$ & $\mathbf{1 . 4 6 0 9}$ & 1.4501 \\
\hline \hline
\end{tabular}


shape feature (cf. Fig. 2). The luminescence spectrum outside the feature [Fig. 3(a)] shows a $1.496 \mathrm{eV}$ InGaAs QW asymmetric and broad full width at half maximum (FWHM) exciton emission. The spectrum is well fitted with a double Gaussian profile. ${ }^{19}$ The highest and smallest energy peaks were attributed to free-exciton (FE) transition and to localized exciton (LE) transition, respectively, which exhibit a slightly lower energy due to potential modulation. A study of InGaAs/InAlAs single QW (Ref. 19) has highlighted that a decrease in QW thickness induces FE and LE FWHM increases. This effect is due to a larger spread of carrier wave function, which leads to higher sensibility to interface and composition fluctuations. In addition, in our case, the FWHM (full width at half maximum $\sim 22.6$ and $60 \mathrm{meV}$, respectively, for FE and LE) are even wider because of large strain variations induced by defects from epitaxy on Si. At
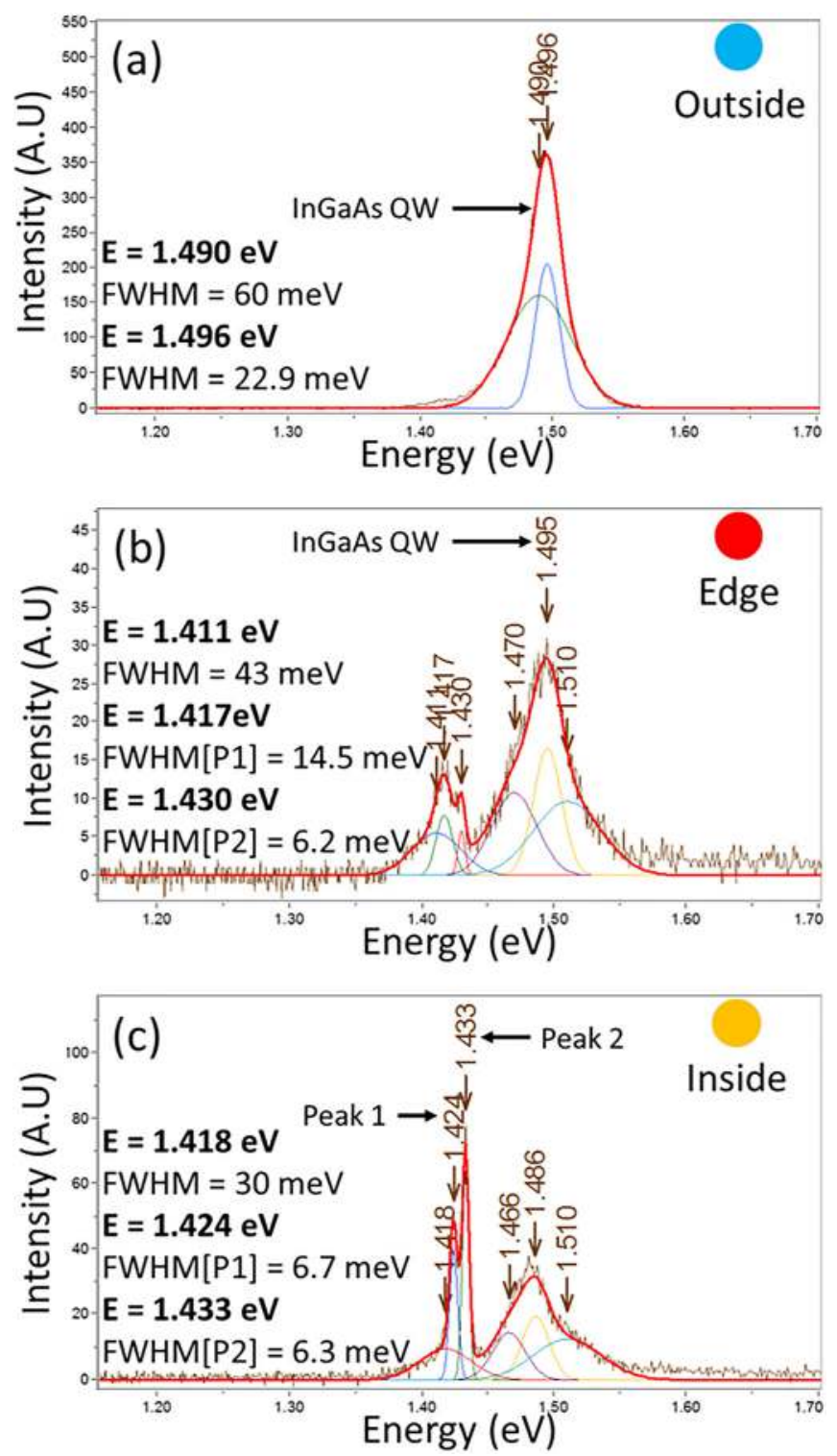

FIG. 3. (Color online) (a) CL spectrum outside of the luminescent circle (in Fig. 2) fitted with a double Gaussian profile due to the anisotropic spectral profile. (b) CL spectrum at the edge of the luminescent circle. (c) CL spectrum inside of the luminescent circle, QW intensity decreased in favor of two narrow peaks (peaks 1 and 2) observed at 1.424 and $1.433 \mathrm{eV}$. the edge of the circular feature [Fig. 3(b)], $1.49 \mathrm{eV}$ FE intensity decreases so much that a very weak emission of GaAs around $1.50 \mathrm{eV}$ can be detected whereas a new emission line at $1.43 \mathrm{eV}$ appears. Inside the feature [cf. Fig. 3(c)], QW FE intensity drops; GaAs is still weakly present whereas a strong double narrow peak appears around $1.43 \mathrm{eV}$ (called peaks 1 and 2, with energies of 1.424 and $1.433 \mathrm{eV}$, respectively) accompanied with a less intense and larger one at lower energy. The origin of this peculiar lower energy doublet, spatially detected as a circular shape, will now be investigated.

In MOCVD growth technique, the most likely defect that can induce an emission at lower energy is the presence of impurities. To explore the nature of impurities, ToF-SIMS profiles were performed on different areas. ${ }^{20}$ Figure 4 presents a qualitative measurement of different elements in the sample. As expected, carbon contaminant has been detected in the first GaAs layer of $30 \mathrm{~nm}$ grown at low temperature on the Si substrate. Moreover, carbon concentration is also found to be very important in both AlAs barriers and buried thick AlAs layer. Some studies have shown that it is difficult to grow AlAs or AlGaAs layers with high purity by MOCVD using trimethyl aluminum (TMAl) as source. ${ }^{21,22}$ Carbon remains highly incorporated due to the formation of Al-carbide during cracking process of TMAl precursor. This explains, in Fig. 4, why a high carbon concentration is mainly detected in AlAs layers $(\mathrm{I} \approx 800$ counts $)$ and less in GaAs layers $(\mathrm{I} \approx 10$ counts $)$ grown at higher temperature. Consequently, in these III-V layers, carbon is mainly present

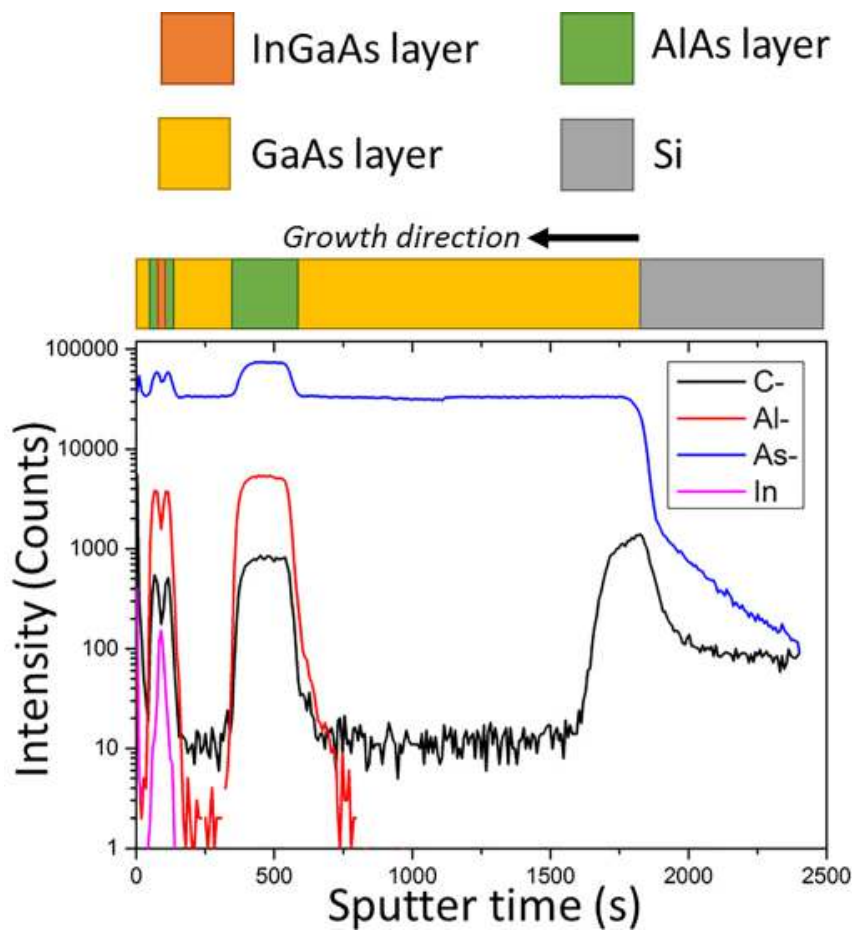

FIG. 4. (Color online) ToF-SIMS depth profile of the sample drawing in Fig. 1. C, Al, As, and In elements are analyzed until silicon substrate. The corresponding layers of analyzed stacking are drawn to facilitate the reading of the chemical profile. The growth direction is horizontal. The profile shows a high $\mathrm{C}$ concentration in AlAs layers, as large as the one of the first GaAs layer grown directly on silicon at low temperature. 
in two ways: (1) a high carbon concentration into AlAs barriers has been detected, so at QW interfaces there is a large carbon amount which could diffuse into QW or interact with confined carriers, and (2) the QW also contains carbon even if the amount is low.

Generally, the value of carbon acceptor binding energy in bulk sample is $26 \mathrm{meV} .{ }^{23}$ However, in the QW case, binding energy depends on the QW width, contaminant position, and strain applied on QW. ${ }^{17,23-25}$ Some previous studies highlight that acceptor binding energy $\left(\mathrm{E}_{\mathrm{B}}\right)$ will increase for oncenter acceptors in a narrow quantum well, ${ }^{17,24}$ whereas it will decrease for on-edge acceptors (or outside) of the QW, i.e., at interfaces (or in barriers). This is possible if excitons remain confined in the QW and impurities are at interfaces or in barriers. In this case, exciton interactions with impurities are lower and, consequently, reduce the binding energy. Furthermore, $\mathrm{E}_{\mathrm{B}}$ in the QW case will decrease when the compressive strain increases. ${ }^{25}$ As InGaAs QW is biaxially strained by GaAs, the binding energy is smaller than the one of GaAs/AlGaAs QW (Refs. 17, 23, and 24) (the most studied case). For different luminescent circles of our sample, we detect a difference energy between the double narrow peak of $\Delta \mathrm{E}=\mid$ Peak $1-$ Peak $2 \mid=11 \pm 2 \mathrm{meV}$. Therefore, to obtain such small $\Delta \mathrm{E}$ value, the most likely possibility is that free-excitons are bound to acceptors located at interfaces or even in barriers. Assuming that peak 2 is free-excitons (FE) emission of the InGaAs QW. Thus, peak 1 is bound excitons (BE) to carbon impurities located at interfaces or in barriers. Consequently, the smallest emission at $1.418 \mathrm{eV}$ could be associated with localized exciton.

As carbon contaminant is present everywhere in the sample, such FE-BE doublet should be detected on the main peak at $1.49 \mathrm{eV}$. Nonetheless, $\mathrm{FE}$ at $1.49 \mathrm{eV}$ has a broad emission line, and its BE emission linked to carbon is not discernable (located on-center or on-edge), whereas for FE at $1.43 \mathrm{eV}$, as its emission is narrow, its $\mathrm{BE}$ emission linked to carbon located on-edge of $\mathrm{QW}\left(\mathrm{E}_{\mathrm{B}}=11 \pm 2 \mathrm{meV}\right)$ is discernable.

The QW certainly also contains carbon located in the QW center. On-center carbon in a $6 \mathrm{~nm}$ QW thickness and taking into account compressive biaxial strain of an InGaAs QW on GaAs will give $\mathrm{E}_{\mathrm{B}}>28 \mathrm{meV}$. $^{23,26}$ Such a line has not been detected on CL spectra for exciton line at $1.49 \mathrm{eV}$ or at $1.43 \mathrm{eV}$. A very low carbon concentration inside QW probably does not allow its detection. Moreover, the probability for a free-exciton to be trapped at an impurity is smaller in QW compared to bulk case ${ }^{24}$ due to shorter life time. The most likely hypothesis is that since interfaces (or barriers) have a higher carbon concentration than in the QW center, then the free-excitons are more likely to be trapped by carbon at interfaces (or in barriers). The intensity of BE linked to on-edge carbon is already low [Fig. 3(c)], so BE emission linked to on-center carbon is not detected in our case. Circular zones exhibit luminescent lines with very small FWHM, indicating a local area with a consistent composition, a stable QW thickness, and good hetero-interfaces.

The sample exhibits two FE energies $(1.49$ or $1.43 \mathrm{eV})$ depending on probed regions. These two QW excitons can be detected on the same pixel due to the electron interaction volume ( $\sim 250 \mathrm{~nm}$ diameter) and diffusion length. They will give a small signal from one or the other depending on the probed region position as shown in Figs. 3(a)-3(c). In addition, from Fig. 2(c), it can be observed that the luminescent shapes are not perfectly circular but tend to be it. This effect is probably also caused by interaction pear and carrier diffusion. As the interaction pear diameter is large, it tends to smooth the true shape of the luminescence area. Concerning carrier diffusion process, it is limited here due to the high defect density. By averaging with the interaction pear, this gives rise to a quasicircular area. The origin of spatially circular shape located at $1.43 \mathrm{eV}$ QW energy will now be investigated.

\section{B. Morphology and precession results}

The structural properties were investigated by STEM and require preparation of thin lamellas. The FIB-SEM tool was used to realize a TEM lamella in cross-section (Fig. 5) that includes the middle of the luminescent circle observed on CL intensity mapping. Using spatial correlation between the CL intensity mapping and STEM [Figs. 5(a) and 5(c)], it can be assumed that TD crossing the QW are present on all samples, inside and outside of the circular shaped areas. Threading dislocations inducing strain variations could be involved in luminescent quenching area located in the middle of luminescent circles. Theoretically, strain can be predicted in 2D layers but not local strain variations induced by the presence of defects. For this reason, N-PED method was used to determine the local strain modification. N-PED strain mappings measure local distortions of the crystal in the plane of the lamella, so here in [001] growth direction $\left(\varepsilon_{\mathrm{zz}}\right)$ [Figs. 6(b) and 6(c)] and in [110] plane direction $\left(\varepsilon_{\mathrm{xx}}\right)$ [Fig. 6(d)]. Although the cross-section does not allow one to analyze all directions of the plane, here mappings confirm the presence of strong strain variations in the area of $1 \mu \mathrm{m}$ on both sides and in the centered area.

In our case, crystal distortion of $\operatorname{In}_{\mathrm{x}} \mathrm{Ga}_{1-\mathrm{x}} \mathrm{As} \mathrm{QW}$ depends on indium quantity and biaxial strain applied by GaAs. The possibility of carbon-induced electronic properties modifications is neglected. Strain $\varepsilon_{z z}$ of $\operatorname{In}_{\mathrm{x}} \mathrm{Ga}_{1-\mathrm{x}} \mathrm{As}$ QW can be expressed with the following relation: ${ }^{7,12}$

$$
\varepsilon_{\mathrm{zz}}=-\frac{2 \mathrm{C}_{12}}{\mathrm{C}_{11}} \varepsilon_{\mathrm{xx}}+\left(1+\frac{2 \mathrm{C}_{12}}{\mathrm{C} 11}\right) \mathrm{f}\left(\mathrm{x}_{\mathrm{In}}\right)
$$

where

$$
\begin{gathered}
\varepsilon_{\mathrm{Xx}}=\frac{\mathrm{a}_{\mathrm{In}_{\mathrm{X}} \mathrm{Ga} a_{1-x} \mathrm{As}}^{\text {real }}-\mathrm{a}_{\mathrm{GaAs}}^{\text {real }}}{\mathrm{a}_{\mathrm{GaAs}}^{\text {real }}}, \\
\mathrm{f}\left(\mathrm{x}_{\mathrm{In}}\right)=\frac{\mathrm{a}_{\mathrm{In}_{\mathrm{x}} \mathrm{Ga} a_{1-x} \mathrm{As}}^{\text {theoreal }}-\mathrm{a}_{\mathrm{GaAs}}^{\text {theoretical }}}{\mathrm{a}_{\mathrm{GaAs}}^{\text {theortical }}} .
\end{gathered}
$$

Here, $a_{\operatorname{In}_{x} \mathrm{Ga}_{1-x} \mathrm{As}}^{\text {theortiol }}$ is the lattice constant (no strained) obtained by Vegard's law for a specific indium quantity $\mathrm{x}_{\mathrm{In}}$ of $\mathrm{In}_{\mathrm{x}} \mathrm{Ga}_{1-\mathrm{x}} \mathrm{As}$; $\mathrm{a}_{\mathrm{GaAs}}^{\text {theoretical }}$ is the lattice constant of GaAs (no strained); and $C_{i j}$ is the elastic stiffness constant of 


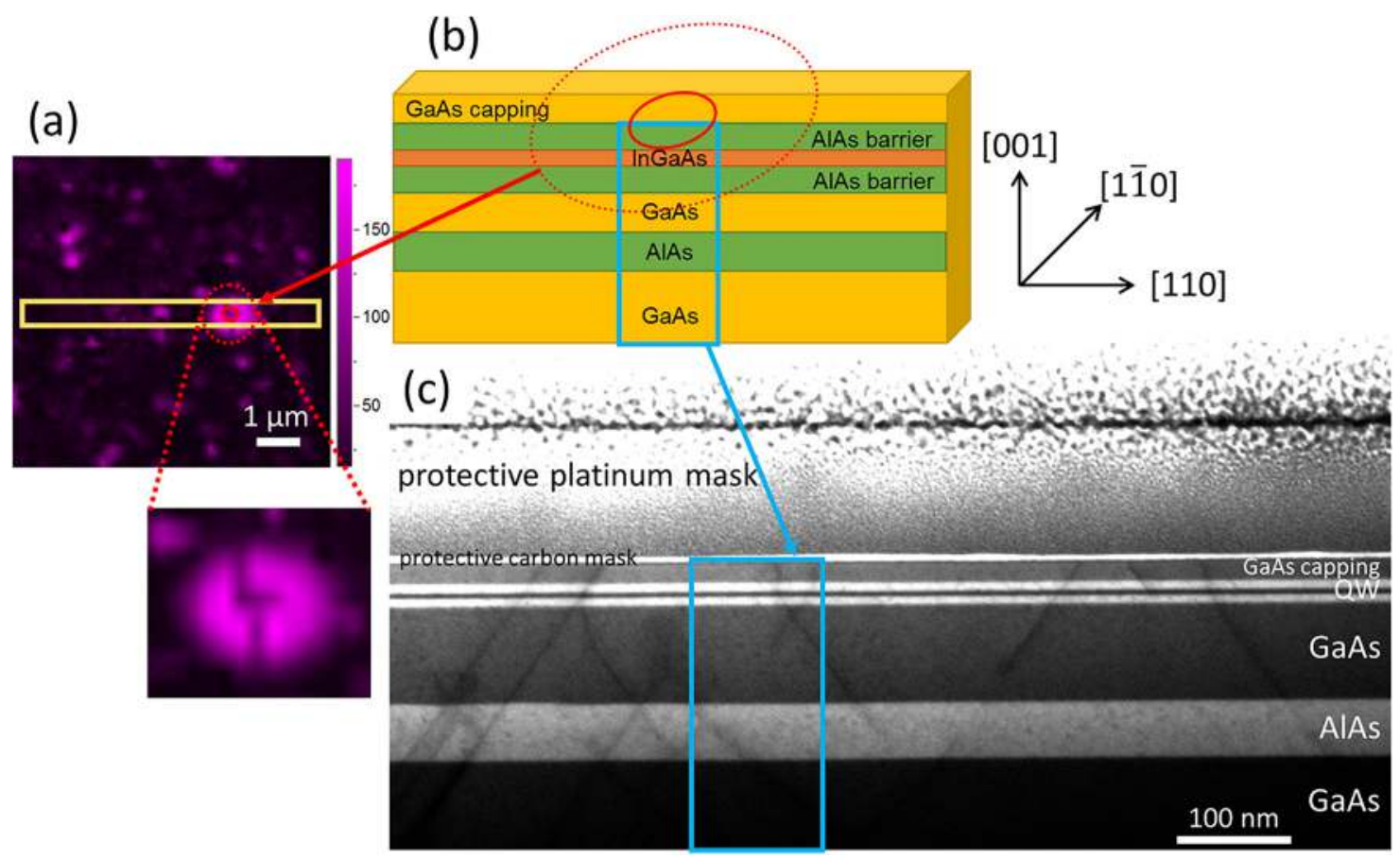

FIG. 5. (Color online) Spatial correlation of the optical and structural properties: (a) CL intensity mapping (top view) shows a local optical emission. Rectangle corresponds to the extracted area comprising the local emission. (b) Drawing of extracted layers used for a cross section lamella. (c) Corresponding STEM image of thin lamella. Rectangle shows the position of local emission.

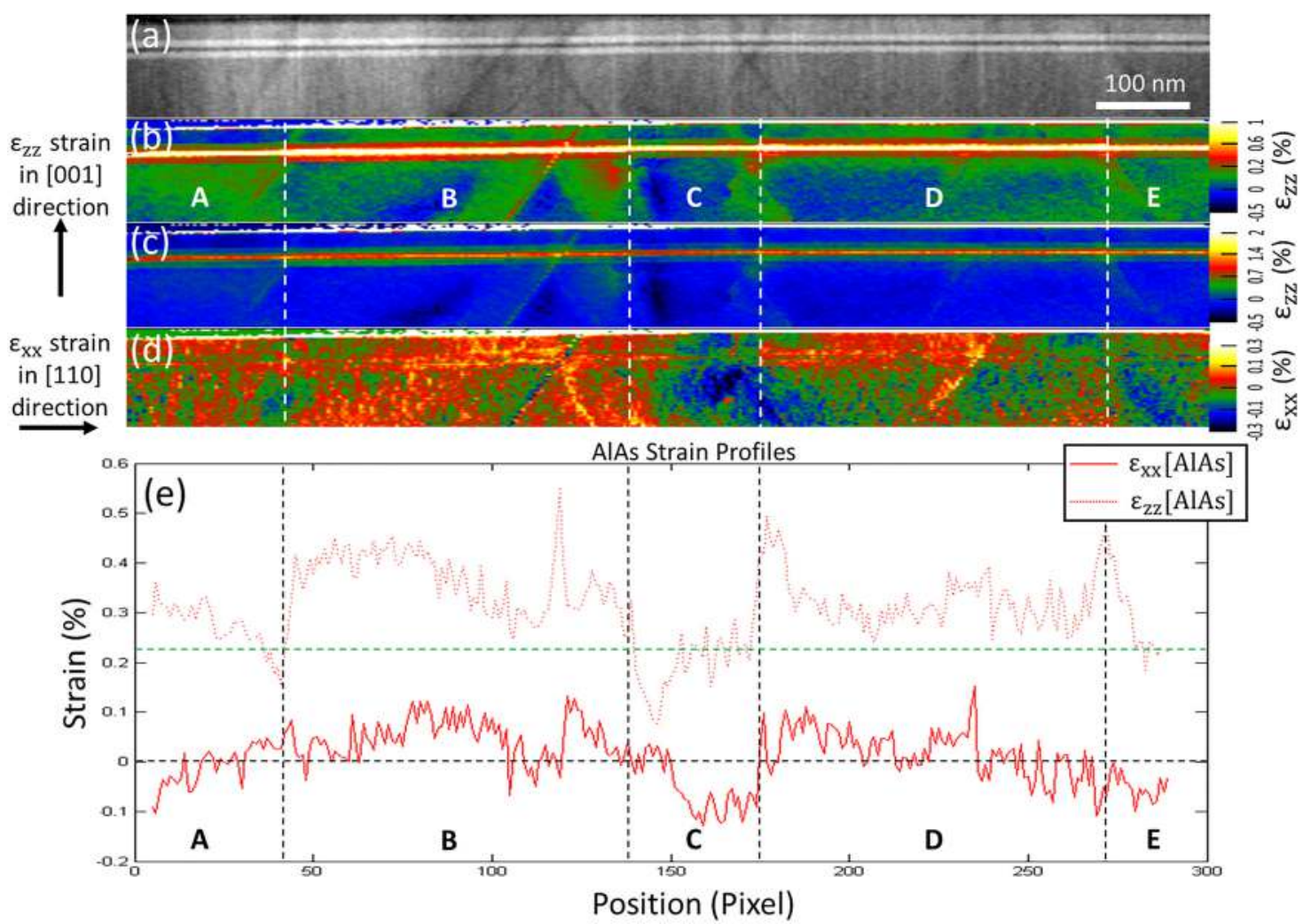

FIG. 6. (Color online) (a) STEM image reconstructed from selected intensity spots of diffraction patterns (precession data acquisition); (b) corresponding: $\varepsilon_{z z}$ strain mapping in [001] direction with scale bare adjusted to observe local strain in GaAs layer, (c) PED $\varepsilon_{z z}$ strain mapping in [001] direction with scale bare adjusted to observe local strain variations in InGaAs QW, and (d) PED strain mapping $\varepsilon_{x x}$ in [110] direction. (e) Integrated $\varepsilon_{x x}$ and $\varepsilon_{z z}$ strain profiles of AlAs barriers along the STEM image. 
$\mathrm{In}_{\mathrm{x}} \mathrm{Ga}_{1-\mathrm{x}}$ As. $\varepsilon_{\mathrm{zz}}$ and $\varepsilon_{\mathrm{xx}}$ correspond to strain in growth [001] and [110] directions, respectively. In the case of a fully relaxed layer, $\mathrm{a}^{\text {real }}=\mathrm{a}^{\text {theoretical }}$ with $\varepsilon_{\mathrm{xx}}=\mathrm{f}\left(\mathrm{x}_{\mathrm{In}}\right)$, so $\varepsilon_{\mathrm{zz}}=\mathrm{f}\left(\mathrm{x}_{\mathrm{In}}\right)$. For a layer in biaxial strain and perfect epitaxy, there is an adaptation of lattice parameter in [110] direction and $\mathrm{a}_{\mathrm{In}_{\mathrm{x}} \mathrm{Ga}_{1-\mathrm{x}} \mathrm{As}}^{\mathrm{rea}}=\mathrm{a}_{\mathrm{GaAs}}^{\mathrm{real}} ;$ thus, $\varepsilon_{\mathrm{xx}}=0$, and finally $\varepsilon_{\mathrm{zZ}}=\left(1+\frac{2 \mathrm{C}_{12}}{\mathrm{C} 11}\right) \mathrm{f}\left(\mathrm{x}_{\mathrm{In}}\right)$. The lattice constant $\mathrm{a}_{\mathrm{In}_{\mathrm{x}} \mathrm{Ga}_{1-\mathrm{x}} \mathrm{As}}^{\text {theoral }}$ $>\mathrm{a}_{\mathrm{GaAs}}^{\text {theoretical }}$ (compressive biaxial strain); therefore, strains become $\varepsilon_{\mathrm{xx}}=0$ and $\varepsilon_{\mathrm{zz}}=\left(1+\frac{2 C_{12}}{C 11}\right) \mathrm{f}\left(\mathrm{x}_{\mathrm{In}}\right)>0$ (tensile strain). The lattice mismatch between AlAs and GaAs is around $\mathrm{f}=0.121 \%$. For a small lattice mismatch, $\mathrm{a}_{\mathrm{AlAs}}=\mathrm{a}_{0}$ and $\varepsilon_{\mathrm{zz}}[\mathrm{AlAs}]=0.0176 \% \approx 0$. This means that no strain induced by the GaAs buffer will affect AlAs. Nevertheless, AlAs barriers surrounding InGaAs has a larger lattice parameter. Thus, $\varepsilon_{\mathrm{zz}}[\mathrm{AlAs}]$ strains tend toward zero near GaAs whereas it increases at InGaAs interfaces.

N-PED technique is mostly used to observe long range strain variations. Unfortunately, it uses a probe size of 2-3 nm. The probe size convolution leads to a degradation of strain resolution for very thin layers as for the case of the InGaAs layer. Consequently, InGaAs values and variations of strain observed on N-PED mappings will not be exploited. For InGaAs strain values, additional measurements by GPA are performed [Fig. 7(b)]. Figures 6(a)-6(e) show STEM image reconstructed, ${ }^{27} \mathrm{~N}$-PED results of the area of interest, and AlAs strain profiles. The GaAs buffer layer is chosen as reference for N-PED strain calculations. Strong strain modifications are used to delimit A, B, C, D, and E areas. Area boundaries are mainly delimited by threading dislocations which cause significant strain variations.

Strain mappings in Figs. 6(b)-6(e) highlight some abnormalities in the AlAs barriers deformation. In Fig. 6(c), important color variations on $\varepsilon_{\mathrm{zz}}[\mathrm{AlAs}]$ are linked to strain modifications and can be observed on the profile in $\varepsilon_{\mathrm{zz}}$ in Fig. 6(e). $\varepsilon_{\mathrm{zz}}[\mathrm{AlAs}]$ is not completely equal to zero as theoretically expected. In Fig. 6(e), strain tendency is $\varepsilon_{\mathrm{zz}}[\mathrm{AlAs}]$ $\approx 0.21 \%$ extracted from Fig. 6(c) for A, C, and E areas. However, B and D areas highlight higher strain $\varepsilon_{\mathrm{zz}}[\mathrm{AlAs}] \approx$ $0.37 \%$. A similar behavior is visible for $\varepsilon_{\mathrm{xx}}[\mathrm{AlAs}]$ in Figs. $6(\mathrm{e})$ and $6(\mathrm{~d})$. In theory, $\varepsilon_{\mathrm{xx}}[\mathrm{AlAs}]=0 \%$. In Fig. 6(e), $\varepsilon_{\mathrm{XX}}[\mathrm{AlAs}]$ tends toward a negative strain for $\mathrm{A}, \mathrm{C}$, and $\mathrm{E}$ areas [extracted from Fig. 6(d)] whereas $\varepsilon_{\mathrm{xx}}[\mathrm{AlAs}]$ tends toward a postive strain for B and D areas. Overall, AlAs strain mappings highlight that $\mathrm{B}$ and $\mathrm{D}$ areas have different strains from others areas (A, C, and E). As AlAs barriers are close to InGaAs QW; thus, their strain modifications could affect InGaAs QW properties. B and D areas are probably linked to the luminescent circle emission. Thereby, N-PED mapping [Fig. 6(e)] highlights that the quenching center of the luminescent circle is located in the $\mathrm{C}$ area.

In the sample, high carbon contamination in AlAs layers was identified by ToF-SIMS (Fig. 4). Carbon doping in AlAs was intensively studied because it produces property modifications. $^{21,28,29}$ Carbon atoms preferably occupy As site. However, the covalent radius of $\mathrm{C}$ atoms is smaller than that of As atoms. At a high doping level $\left(10^{18}-10^{19}\right.$ $\mathrm{cm}^{-3}$ ), it induces lattice contraction and introduces a tensile strain in the C-doped AlAs layers. It has been found that GaAs/AlGaAs interface presents a higher carbon contamination trapped during growth. ${ }^{30,31}$ Accumulation of carbon on GaAs layer near GaAs/AlGaAs interface would come from the difference of solubility between both materials. In Figs. 6(c), B and D strain areas highlight a GaAs/ AlAs interface with steep profiles which could be induced by carbon accumulation. The origin of these steep profiles comes from a small decrease in $\varepsilon_{\mathrm{zz}}$ strain [shown by arrows in Fig. 7(a)]. This drop of $\varepsilon_{\text {zz }}$ involves a sudden lattice contraction, coherent if GaAs/AlAs interfaces present an intermediate layer such as AlGaAs:C with a high carbon concentration ( $\left.\mathrm{a}_{\mathrm{AlGaAs}: \mathrm{C}}<\mathrm{a}_{\mathrm{AlAs}} \mathrm{C}\right)$. The strain drop occurs at GaAs/AlAs interface only. Indeed, Figs. 6(b)-6(e) show a positive strain increase in barriers of both $\varepsilon_{\mathrm{xx}}$ and $\varepsilon_{\mathrm{zz}}$. An AlAs positive strain value is possible for particular cases with higher carbon concentration. ${ }^{32}$ This means that a higher carbon concentration inside the barriers is probably present for B and D areas. These local modifications of B and $\mathrm{D}$ areas could explain the luminescent circle emission. The B and D areas seem to be locally delimited by threading dislocations. The C-enrichment in some parts of the sample seems to be induced by particular strain modifications caused by dislocations in the volume. Dislocations are already present during growth process and induce locally significant deformations which are able to modify species concentration. As a reminder, cross-section strain mapping does not allow to analyze a single luminescent circle in all directions of the plane. Thus, extrapolations
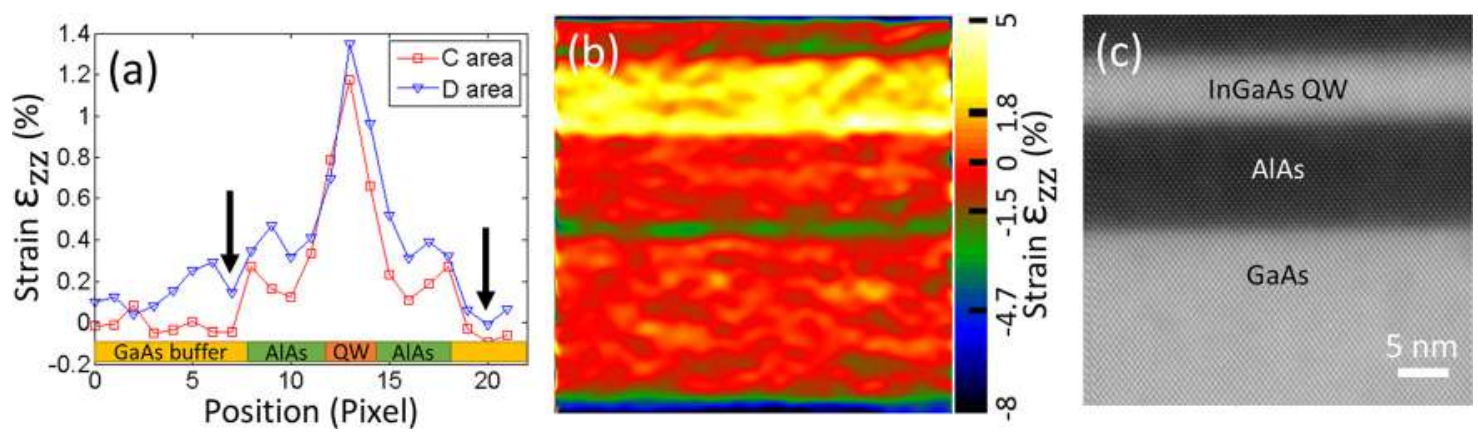

FIG. 7. (Color online) (a) $\varepsilon_{z z}$ strain profiles extracted from C and D areas of the region indicated in Fig. 6(c). (b) $\varepsilon_{z z}$ strain obtained by GPA. (c) Corresponding HR-STEM image associated with Fig. 7(b). 
are done on tendencies shown in two-dimensions obtained by N-PED and GPA.

Previously, the double peak has been associated with BE and $\mathrm{FE}$ InGaAs QW emission, but the origin of such low free-exciton energy $(\mathrm{FE}=1.433 \mathrm{eV})$ inside luminescent circle has not been fully explained. Four main ways are able to shift an emission energy: impurities, thickness, strain, and indium content. First hypothesis: impurities incorporated in the QW center are not considered here because no increase in binding energy $(\Delta \mathrm{E}=\mathrm{FE}-\mathrm{BE} \geq 28 \mathrm{meV})$ has been detected $^{17,24}$ as previously mentioned. Second hypothesis: an increase in QW thickness leads to a decrease in quantum confinement. However, STEM and TEM images show a uniform thickness of QW. Third and fourth hypotheses: a strain reduction or an increase in indium concentration could also lead to a shift in energy. One of these two hypotheses is probably responsible for the decrease in FE energy. Unfortunately, some previous studies highlight the complexity to dissociate effects of strain and In fluctuations. ${ }^{33-35}$ Figure 8 (a) shows $\varepsilon_{\mathrm{zz}}$ strain variations of InGaAs on GaAs as function of indium content determined using Eq. (2) for different relaxation rates from completely biaxial strained $(0 \%)$ to fully relaxed cases (100\%). Unfortunately, as mentioned earlier, N-PED strain values for InGaAs QW are undervalued due to probe size convolution. To reach atomic resolution, additional GPA measurements were performed. Figure 7(b) shows GPA measurements of the D area, with an extracted strain $\varepsilon_{\mathrm{zz}(\max )}[\mathrm{InGaAs}]=2.3 \%$. By reporting this value in Fig. 8(a), such high strain can be obtained only for an InGaAs layer biaxially strained on GaAs with In $\approx 16.5 \%$. We notice that, in the range of In $\approx 11 \% \pm 1.5 \%$ previously determined by CL, no such high strain value can be determined even in partially relaxed cases. Using NextNano software, the FE emission energies of InGaAs QW $(6 \mathrm{~nm})$ are simulated for various indium content. Results of the simulation are presented in Fig. 8(b). Both limit cases have been plotted in Fig. 8(b) showing FE of a biaxially strained QW in red and a fully relaxed QW in blue. By relating the previously determined indium content (In $\approx 16 \%$ in Fig. $8(\mathrm{~b})$, a free exciton energy of $1.43 \mathrm{eV}$ is obtained and corresponds to the exciton energy measured in luminescent circles. In addition, $\mathrm{FE}_{\mathrm{In}} \approx 0.16 \approx 1.43 \mathrm{eV}$ takes place only when on-edge-carbon-bound-exciton exists. Therefore, it seems to indicate that the significant increase in carbon concentration into AlAs barriers is also directly related to the local increase in indium concentration.

N-PED shows that the quenching center of the luminescent circle corresponds to the $\mathrm{C}$ area in Figs. 6(c)-6(e). This area has a smaller strain than B and D areas. During growth on $\mathrm{Si}$, the III-V layers are subjected to high strains. Nevertheless, some parts could be subjected to higher strains than others and locally layers should have to release it with an array of dislocations around this area. In particular cases, these areas promoted $\mathrm{C}$ - and In-enrichment. At the end of the growth, a little compressive strain area still subsists (the center) and is surrounded by a larger less strained area with C- and In-enrichment (higher positive AlAs strain values). As the center is more strained than around, its gap is higher.
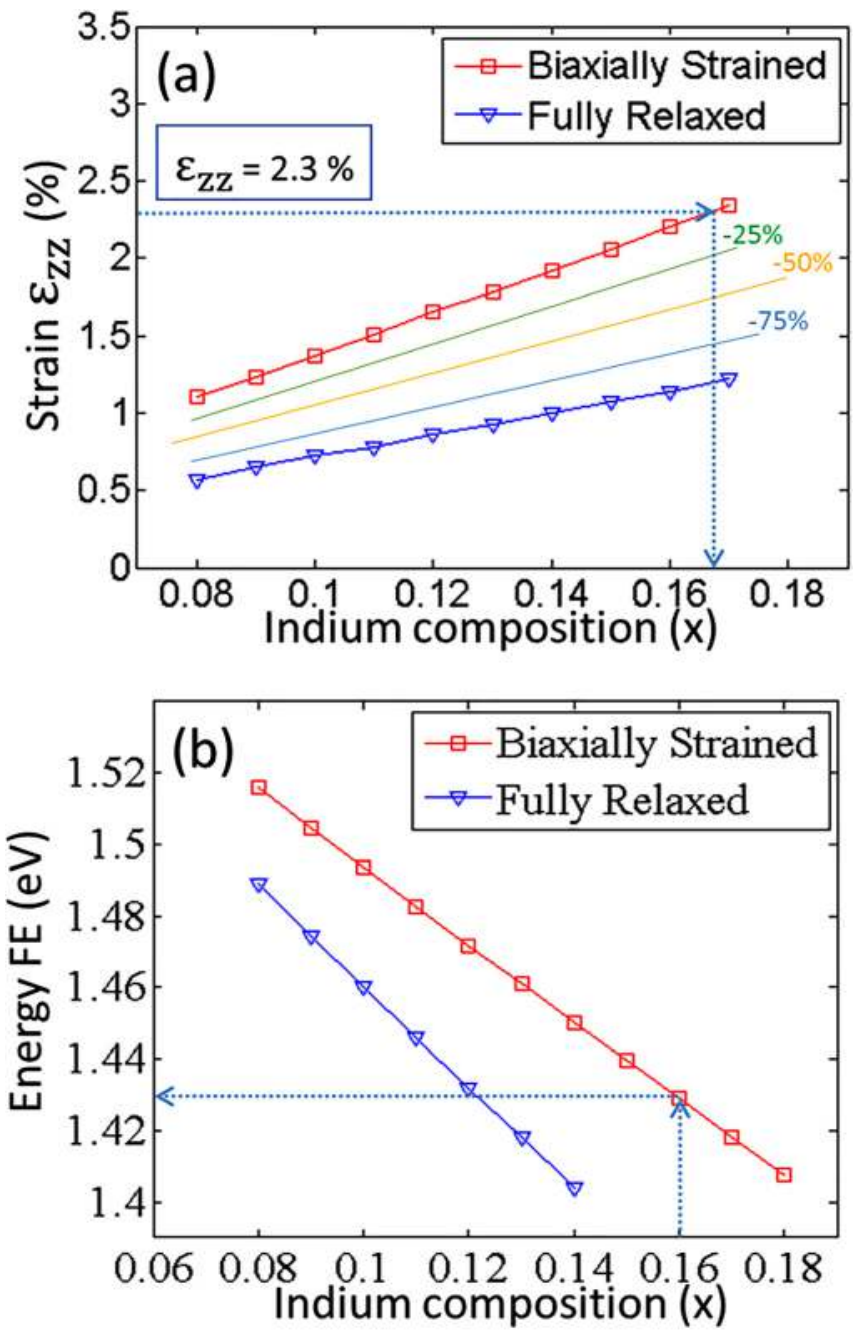

FIG. 8. (Color online) (a) Amount of theoretical indium quantity as a function of $\varepsilon_{z z}\left[\operatorname{In}_{\mathrm{x}} \mathrm{Ga}_{1-\mathrm{x}} \mathrm{As}\right]$ for different rates of strain reduction. (b) Variation of the free exciton (FE) emission energy as a function of indium quantity incorporated in the $\operatorname{In}_{\mathrm{x}} \mathrm{Ga}_{1-\mathrm{x}} \mathrm{As} \mathrm{QW}$.

Lower gap is more favorable for carriers' recombination so the center seems always quenching the luminescence.

Consequently, it has been shown that $1 \mu \mathrm{m}$ luminescent areas highlight strong strain modification (higher positive strain values for $\varepsilon_{\mathrm{xx}}[\mathrm{AlAs}]$ and $\left.\varepsilon_{\mathrm{zz}}[\mathrm{AlAs}]\right)$ characteristics of local carbon enrichment in the AlAs barriers. Local C-enrichments seem to be promoted by local strain modifications around threading dislocations. These strain modifications cause at the same time a C-enrichment in AlAs barriers and an In-enrichment in the InGaAs QW. Therefore, the double peak emission of the luminescent circles is identified as $\quad \mathrm{FE}_{\mathrm{In}} \approx 0.16=1.433 \mathrm{eV}$ and on-edge-carbon-BE $\mathrm{In} \approx 0.16$ $=1.424 \mathrm{eV}$ emissions. In regards to the FWHM and the constant energy of these two peaks, areas of higher strain have a more stable In-composition and constant strain, all promoted by local C-enrichment in AlAs barriers.

\section{CONCLUSIONS}

For the direct III-V heteroepitaxy on Si substrate by MOCVD, CL investigation of InGaAs QW highlights 
specific double peak emission characterized by a constant energy emission lying on specific luminescence shapes of $1 \mu \mathrm{m}$ diameter. The double peak emission is separated by a low binding energy $\mathrm{E}_{\mathrm{B}}=11 \pm 2 \mathrm{meV}$. ToF-SIMS experiments have detected the presence of a high amount of carbon in AlAs barriers. Carbon is known to remain incorporated in AlAs growth with TMAL precursor in MOCVD growth. Dislocations are highlighted by (S)TEM images. Their presence during the growth process induces locally significant strain variations delimited by threading dislocations. Luminescent circular shapes present different strain characteristics. It is highlighted that they correspond to a higher strain area surrounding a less strained area in AlAs barriers and InGaAs QW. These strain modifications can explain the increases of $\mathrm{C}$ concentration in AlAs barriers and of In concentration in InGaAs QW, thanks to a local modification of In and $\mathrm{C}$ incorporation rate. This leads to local increases of In concentration up to $16 \%$ emitting at a lower energy $(1.433 \mathrm{eV})$ as proved by theoretical calculations. Therefore, the double peak corresponds to free-exciton and to on-edgecarbon-acceptor-bound to exciton emissions of QW in regions of higher strain and are linked to increases of $\mathrm{C}$ and In concentrations in barriers and QW, respectively.

The tendency of III-V heteroepitaxy on $\mathrm{Si}$ is to reduce the buffer layer thickness and to trap most defects therein. This study highlights the impact of strain fluctuations induced by dislocations on the nanostructure homogeneity intended for opto and electronic devices as optical emitters or transistors. It leads to interest in strain variations contained in buffer resulting from modifications in a QW structure. Finally, it has been shown in a peculiar case how strain modifications enhanced by the presence of threading dislocations can modify the local optical properties of an InGaAs QW embedded between two AlAs barriers, in particular, how the III-V layers are chemically modified.

\section{ACKNOWLEDGMENTS}

This work has been partially supported by the LabEx Minos ANR-10-LABX-55-01. This work made use of the nanocharacterization platform (PFNC) facilities at Minatec. This project has received funding from the Electronic Component Systems for European Leadership Joint Undertaking under Grant Agreement No. 692527 (3DAM). The authors are thankful to B. Masenelli for his fruitful discussions.

${ }^{1}$ R. R. Bradley, J. A. Beswick, T. B. Joyce, P. D. Hodson, P. Kightley, R. I. Taylor, D. J. Stirland, and R. J. M. Griffiths, Vacuum 40, 339 (1990).

${ }^{2}$ S. C. Jain, M. Willander, and H. Maes, Semicond. Sci. Technol. 11, 641 (1996).
${ }^{3}$ R. Alcotte et al., APL Mater. 4, 046101 (2016).

${ }^{4}$ M. Martin et al., Appl. Phys. Lett. 109, 253103 (2016).

${ }^{5}$ A. A. Marmalyuk, O. I. Govorkov, A. V. Petrovsky, D. B. Nikitin, A. A. Padalitsa, P. V. Bulaev, I. V. Budkin, and I. D. Zalevsky, J. Cryst. Growth 237, 264 (2002).

${ }^{6}$ Yu. A. Goldberg and N. M. Schmidt, Handbook Series on Semiconductor Parameters (World Scientific, Singapore, 1999).

${ }^{7}$ H. Asai and K. Oe, J. Appl. Phys. 54, 2052 (1983).

${ }^{8}$ I. Pelant and J. Valenta, Luminescence Spectroscopy of Semiconductors (Oxford University, Oxford, United Kingdom, 2012).

${ }^{9}$ J. L. Rouvière, A. Mouti, and P. Stadelmann, J. Phys. Conf. Ser. 326, 012022 (2011).

${ }^{10}$ S. David et al., APL Mater. 4, 056102 (2016).

${ }^{11}$ J.-L. Rouviere, A. Béché, Y. Martin, T. Denneulin, and D. Cooper, Appl. Phys. Lett. 103, 241913 (2013).

${ }^{12}$ D. Cooper, N. Bernier, J.-L. Rouvière, Y.-Y. Wang, W. Weng, A. Madan, S. Mochizuki, and H. Jagannathan, Appl. Phys. Lett. 110, 223109 (2017).

${ }^{13}$ M. J. Hÿtch, E. Snoeck, and R. Kilaas, Ultramicroscopy 74, 131 (1998).

${ }^{14}$ J. L. Rouvière and E. Sarigiannidou, Ultramicroscopy 106, 1 (2005).

${ }^{15}$ J. L. Rouviere, "The use of the geometrical phase analysis to measure strain in nearly periodic images," in Microscopy of Semiconducting Materials 2007, Springer Proceedings in Physics, edited by A. G. Cullis and P. A. Midgley (Springer, Dordrecht, 2008), Vol 120.

${ }^{16}$ A. Ajay, C. B. Lim, D. A. Browne, J. Polaczyński, E. Bellet-Amalric, J. Bleuse, M. I. den Hertog, and E. Monroy, Nanotechnology 28, 405204 (2017).

${ }^{17} \mathrm{P}$. Harrison, Quantum Wells, Wires and Dots, 3rd ed. (Wiley, West Sussex, England, 2010).

${ }^{18}$ V. K. Yang, S. M. Ting, M. E. Groenert, M. T. Bulsara, M. T. Currie, C. W. Leitz, and E. A. Fitzgerald, J. Appl. Phys. 93, 5095 (2003).

${ }^{19}$ Y. Wang et al., Nanoscale Res. Lett. 12, 1 (2017).

${ }^{20}$ H. Schwenke, J. Knoth, L. Fabry, S. Pahlke, R. Scholz, and L. Frey, J. Electrochem. Soc. 144, 3979 (1997).

${ }^{21}$ S. Hirose, A. Yoshida, M. Yamaura, N. Kano, and H. Munekata, J. Mater. Sci. Mater. Electron. 11, 7 (2000).

${ }^{22}$ N. Kobayashi and T. Makimoto, Jpn. J. Appl. Phys., Part 2 24, L824 (1985).

${ }^{23}$ K. Sumino, Defect Control in Semiconductors (Elsevier Science, NorthHolland, 1989).

${ }^{24}$ O. Holtz, Impurities Confined in Quantum Structures (Springer, Berlin, Germany, 2013).

${ }^{25}$ J. P. Loehr, Y. C. Chen, D. Biswas, P. Bhattacharya, and J. Singh, Appl. Phys. Lett. 57, 180 (1990).

${ }^{26}$ W. T. Masselink, Y.-C. Chang, and H. Morkoc, Phys. Rev. B 32, 5190 (1985).

${ }^{27}$ J. L. Rouviere, Y. Martin, N. Bernier, M. Vigouroux, D. Cooper, and J. M. Zuo, Microsc. Microanal. 21, 2209 (2015).

${ }^{28}$ S. Sekigughi and T. Miyamoto, Jpn. J. Appl. Phys., Part 1 36, 2638 (1997).

${ }^{29}$ N. Watanabe, T. Nittono, and H. Ito, J. Cryst. Growth 145, 929 (1994).

${ }^{30}$ D. H. Zhang and C. Y. Li, Superlattices Microstruct. 24, 119 (1998).

${ }^{31}$ P. M. Petroff, R. C. Miller, A. C. Gossard, and W. Wiegmann, Appl. Phys. Lett. 44, 217 (1984).

${ }^{32}$ J. D. MacKenzie, C. R. Abernathy, S. J. Pearton, and S. N. G. Chu, Appl. Phys. Lett. 66, 1397 (1995).

${ }^{33}$ K. Mukherjee, D. A. Beaton, A. Mascarenhas, M. T. Bulsara, and E. A. Fitzgerald, J. Cryst. Growth 392, 74 (2014).

${ }^{34}$ P. Roura, A. Vila, J. Bosch, M. López, A. Cornet, J. R. Morante, and D. I. Westwood, J. Appl. Phys. 82, 1147 (1997).

${ }^{35}$ K. Rammohan, D. H. Rich, R. S. Goldman, J. Chen, H. H. Wieder, and K. L. Kavanagh, Appl. Phys. Lett. 66, 869 (1995). 